\title{
A simple inductive measure analysis for cardinals under the Axiom of Determinacy
}

\author{
Stefan Bold, Benedikt Löwe
}

\begin{abstract}
In this paper, we give a thorough and basic introduction to the main techniques dealing with computation of cardinals under the Axiom of Determinacy by measure analyses. As an application, we give a simple inductive measure analysis (without invoking Jackson's "description theory") that allows the computation of further Jónsson cardinals.
\end{abstract}

The Axiom of Determinacy AD is a game-theoretic statement expressing that all infinite two-player perfect information games with a countable set of possible moves are determined, i.e., admit a winning strategy for one of the players. The restriction to countable sets of possible moves makes $A D$ essentially a statement about real numbers and sets of real numbers, and traditionally it has been investigated by descriptive set theorists.

As a consequence, it comes as a surprise to see that AD has strikingly peculiar consequences for the combinatorics on uncountable cardinals such as $\aleph_{\omega}$ : for example, the Axiom of Determinacy implies that every algebra on $\aleph_{\omega}$ has a proper subalgebra of cardinality $\aleph_{\omega}$. How can an axiom that talks about the existence of real numbers have consequences for a cardinal like $\aleph_{\omega}$ ?

There are (at least) two parts to this answer: First of all, under the assumption of $A D$, a cardinal like $\aleph_{\omega}$ is not so far removed from the reals as we like to think. If we let

$$
\Theta:=\sup \{\alpha ; \text { there is a surjection from } \mathbb{R} \text { onto } \alpha\},
$$

then it is a consequence of Moschovakis' Coding Lemma (observed by H. Friedman and Solovay $\left.{ }^{[1]}\right)$ that $\Theta$ is far bigger than $\aleph_{\omega}$. As $\Theta$ is the reflection of the real numbers in the ordinals, (part of) the combinatorial theory of cardinals $\kappa<\Theta$ is affected by the theory of the reals. For instance, using the surjection $\pi: \mathbb{R} \rightarrow \kappa$, we can translate statements of the first-order theory of $\kappa$ into statements of secondorder arithmetic (with parameter $\pi$ ).

But as the second part of the answer, we can go beyond this vague statement and make it more concrete. By a general argument of Solovay, we can connect games to the existence of ultrafilters on cardinals $\kappa<\Theta$ : Using a surjection $\pi: \mathbb{R} \rightarrow \kappa$,

2000 Mathematics Subject Classification. Primary 03E60, 03E05; Secondary 03E10.

Both authors were funded by a DFG-NWO Bilateral Cooperation Grant (DFG KO 1353/3-1; NWO DN 61-532)

${ }^{[1]}$ For details, $c f$. [Ka94, Exercises $\left.28.16 \& 28.17\right]$. 
we can play a usual game (with at most countably many moves) and interpret it (by coding) as a game on $\kappa$. These games are called Solovay games. Solovay developed a general technique of associating Solovay games $\mathrm{G}_{A}$ to subsets $A \subseteq \kappa$ in such a way that

$$
\mathcal{S}_{\kappa}:=\left\{A \subseteq \kappa ; \text { Player I has a winning strategy in } \mathrm{G}_{A}\right\}
$$

defines an ultrafilter on $\kappa$. The first application of this technique was Solovay's famous proof of the measurability of $\aleph_{1}$ under AD (for a proof, $c f$. [Ka94, Theorem 28.2]). Moreover, Solovay's proof tells us that the $\omega_{1}$-complete ultrafilter on $\aleph_{1}$ is the club filter $\mathcal{C}_{\omega_{1}}^{\omega}$.

An early extension of Solovay's technique to yield strong combinatorial results for uncountable cardinals was Martin's 1973 theorem that established the strong partition property ${ }^{[2]}$ of $\aleph_{1}$ under AD [Ka94, Theorem 28.12]. This connected the theory of $A D$ with the study of infinite exponent partition relations and opened many other possibilities for analysing the structure of uncountable cardinals under AD. Kleinberg (1977) proved that strong partition cardinals $\kappa$ together with a normal measure $\mu$ on $\kappa$ generate a sequence $\left\langle\kappa_{n}^{\mu} ; n \geq 1\right\rangle$ of partition cardinals (called a Kleinberg sequence; cf. Theorem 3), and computed the sequence derived from the strong partition property of $\aleph_{1}: \kappa_{n}^{\mathcal{C}}=\aleph_{n}$. This result yields the mentioned combinatorial property of $\aleph_{\omega}$.

Nowadays, we know much more about infinitary combinatorics under AD than in 1977, and it was mainly the work of Steve Jackson [Ja88, Ja99] that gave us many more strong partition cardinals and normal measures on cardinals below $\aleph_{\varepsilon_{0}}$. However, a uniform analysis of all cardinals in terms of measures is still a desideratum. Jackson and Khafizov [JaKh $\infty$ ] have provided a full analysis for cardinals $\kappa<\boldsymbol{\delta}_{5}^{1}=\aleph_{\omega^{\omega} \omega}{ }^{\omega}+1$ which has been used by the second author in [Lö02] to compute more Kleinberg sequences, but so far, there is no uniform way of giving a full measure analysis.

In this paper, we give a survey of measure analyses under AD in Section 1 (based on work of Jackson and the second author). In Section 2, we discuss the general theory of order measures that form the theoretical foundations of the main result of this paper. Sections 1 and 2 are to be understood as a survey of techniques that are either folklore results or essentially due to Jackson. Though none of the results of these sections are original, the presentation is new and much more thorough and detailed than in any existing publication. Our goal was not just to provide the techniques needed for our proof, but to give a general introduction for the uninitiated reader who has not read any of Jackson's papers.

Then, in Section 3, we give a simple inductive argument (that avoids Jackson's description theory) for a measure analysis with just two measures that reaches the first $\omega^{2}$ cardinals after a strong partition cardinal. As an application, we give the computation of the Kleinberg sequences of the $\omega_{1}$-cofinal measure on odd projective ordinals in Section 4. Using further results of Kleinberg's, we answer an open question from [Lö02].

${ }^{[2]} C f$. Section 1 for definitions. 


\section{Mathematical Background}

In this paper, we shall be working in the system $Z F+D C+A D$. Very few results need the full strength of AD: it is enough for many results to work in ZF + DC, assume that $\kappa$ is a strong partition cardinal and have enough choice available to make sure that the club filter on $\kappa$ is $\sigma$-complete.

1.1. Basics. We define the iterated successor operation on cardinals $\kappa$ as follows by transfinite recursion:

- $\kappa^{(0)}=\kappa$,

- $\kappa^{(\alpha+1)}=\left(\kappa^{(\alpha)}\right)^{+}$for all ordinals $\alpha$, and

- $\kappa^{(\lambda)}=\bigcup\left\{\kappa^{(\alpha)} ; \alpha \in \lambda\right\}$ for limit ordinals $\lambda$.

Let $\kappa$ be a cardinal. We shall say that $\kappa$ has the strong partition property if the partition relation $\kappa \rightarrow(\kappa)^{\kappa}$ holds, i.e., if for every partition of $[\kappa]^{\kappa}$ into two blocks there is a homogeneous set of order type $\kappa$. Note that the strong partition property cannot hold for any cardinal if we assume the Axiom of Choice AC: by a result of Erdős and Rado ( $c f$. [Ka94, Proposition 7.1]) any partition relation with infinite exponents violates AC.

Let us define the $\lambda$-cofinal filter $\mathcal{C}_{\kappa}^{\lambda}$ as the filter generated by the $\lambda$-closed unbounded sets in $\kappa$, i.e.,

$$
A \in \mathcal{C}_{\kappa}^{\lambda}: \Longleftrightarrow \text { there is a club set } C \subseteq \kappa \text { such that }\{\alpha \in C ; \operatorname{cf}(\alpha)=\lambda\} \subseteq A .
$$

Clearly, $\mathcal{C}_{\omega_{1}}^{\omega}$ is the ordinary club filter on $\omega_{1}$. As usual, we call a $\sigma$-complete ultrafilter a measure, and we call it normal if it is closed under diagonal intersection. If $\mu$ is a normal filter on $\omega_{1}$, then it must contain the club filter $\mathcal{C}_{\omega_{1}}^{\omega}$. If $\mu$ is a measure on $\kappa$ and $\alpha$ is an ordinal, then (because of DC) the ultrapower $\alpha^{\kappa} / \mu$ is wellfounded and thus isomorphic to an ordinal. We identify it with its Mostowski collapse.

The strong partition property of $\kappa$ implies the existence of many concrete measures on $\kappa$, as the following theorem of Kleinberg shows:

Theorem 1. Let $\kappa$ be a cardinal with the strong partition property and $\lambda<\kappa$ a regular cardinal. Then $\mathcal{C}_{\kappa}^{\lambda}$ is a normal measure. In addition, if $\kappa$ is not weakly Mahlo, then these are the only normal ultrafilters on $\kappa$.

Proof. [Ka94, Theorem 28.10 \& Exercise 28.11].

In other words, the strong partition property of $\kappa$ not only gives the existence of measures, but in our case (our cardinals will be below $\aleph_{\varepsilon_{0}}$ and thus not weakly Mahlo) also a structured pattern of all of the normal measures on $\kappa$ (indexed by the regular cardinals below $\kappa$ ).

In addition, the strong partition property also connects to other combinatorial properties that are well known from usual (AC-) combinatorial set theory: A cardinal $\kappa$ is called a Jónsson cardinal if the partition relation $\kappa \rightarrow[\kappa]_{\kappa}^{<\omega}$ holds, i.e., for every partition of $[\kappa]^{<\omega}$ into $\kappa$ blocks there is a set $H$ of order type $\kappa$ with the property that $[H]^{<\omega}$ doesn't meet all blocks. ${ }^{[3]}$

A cardinal $\kappa$ is called a Rowbottom cardinal if for all $\lambda<\kappa$ the partition relation $\kappa \rightarrow[\kappa]_{\lambda,<\omega}^{<\omega}$ holds, i.e., for every partition of $[\kappa]^{<\omega}$ into $\lambda$ blocks there is

${ }^{[3]}$ This is equivalent to saying that every algebra (in the sense of universal algebra) on $\kappa$ has a proper subalgebra of size $\kappa$. [Co96] is a nice survey of the algebraic side of the Jónsson property. 
a set $H$ of order type $\kappa$ with the property that $[H]^{<\omega}$ meets only countably many blocks.

DEFINITION 2. Let $\kappa$ be a strong partition cardinal and $\mu$ a normal measure on $\kappa$. We then define a sequence of wellordered structures $\left\langle\kappa_{n}^{\mu} ; n \leq \omega\right\rangle$ as follows:

- $\kappa_{1}^{\mu}:=\kappa$,

- $\kappa_{n+1}^{\mu}:=\left(\kappa_{n}^{\mu}\right)^{\kappa} / \mu$, and

- $\kappa_{\omega}^{\mu}:=\sup \left\{\kappa_{n}^{\mu} ; n \in \omega\right\}$.

This sequence is called the Kleinberg sequence derived from $\mu$.

THEOREM 3. Let $\kappa$ be a strong partition cardinal and $\mu$ be a normal measure on $\kappa$. Then

(1) $\kappa_{1}^{\mu}$ and $\kappa_{2}^{\mu}$ are measurable,

(2) for all $n \geq 2, \operatorname{cf}\left(\kappa_{n}^{\mu}\right)=\kappa_{2}^{\mu}$,

(3) $\kappa_{n}^{\mu}$ is a Jónsson cardinal, and

(4) $\kappa_{\omega}^{\mu}$ is a Rowbottom cardinal.

Moreover, if $\kappa_{2}^{\mu}=\kappa^{+}$, then $\kappa_{n+1}^{\mu}=\left(\kappa_{n}^{\mu}\right)^{+}$for all $n \in \omega$, and $\kappa_{2}^{\mu} \rightarrow\left(\kappa_{2}^{\mu}\right)^{\alpha}$ for all $\alpha<\kappa_{2}^{\mu}$.

Proof. Cf. [K177].

1.2. The Axiom of Determinacy. Let us connect the theory of strong partition cardinals to the Axiom of Determinacy AD. The Axiom of Determinacy implies a certain fragment of $A C$ that will be useful later:

LemmA 4. Let $\kappa<\Theta$. Then $\mathrm{AC}_{\omega}\left(\kappa^{\kappa}\right)$ holds, i.e., every countable family of nonempty subsets of $\kappa^{\kappa}$ has a choice function.

Proof. Since $\kappa<\Theta$, Moschovakis' Coding Lemma (cf. [Ka94, Theorem 28.15]) yields a surjection $\pi: \omega^{\omega} \rightarrow \kappa^{\kappa}$. Suppose that for each $i \in \omega$, the set $X_{i} \subseteq \kappa^{\kappa}$ is nonempty. Then let $\mathrm{G}_{\left\langle X_{i} ; i \in \omega\right\rangle}$ be the following game:

Player I plays a natural number $n$, Player II then plays in $\omega$ moves a sequence $y \in \omega^{\omega}$. Player II wins $\mathrm{G}_{\left\langle X_{i} ; i \in \omega\right\rangle}$ if $\pi(y) \in X_{n}$. Clearly, player I cannot have a winning strategy, so by AD, player II has one. But a winning strategy for player II is a choice function for the family $\left\langle X_{i} ; i \in \omega\right\rangle$.

In descriptive set theory, definable analogues of the cardinal $\Theta$ have been investigated, the so-called projective ordinals

$$
\boldsymbol{\delta}_{n}^{1}:=\sup \left\{\xi ; \xi \text { is the length of a prewellordering of } \omega^{\omega} \text { in } \boldsymbol{\Delta}_{n}^{1}\right\} .
$$

In the early $1980 \mathrm{~s}$, a lot of combinatorial consequences of $\mathrm{AD}$ for the projective ordinals were known, among them the following:

TheOREM 5. Let $n$ be a natural number. Then:

(1) (Kunen, Martin 1971) $\boldsymbol{\delta}_{2 n+2}^{1}=\left(\boldsymbol{\delta}_{2 n+1}^{1}\right)^{+}$,

(2) (Kechris 1974) $\boldsymbol{\delta}_{2 n+1}^{1}$ is the cardinal successor of a cardinal of cofinality $\omega$,

(3) (Martin, Kunen 1971) all $\boldsymbol{\delta}_{n}^{1}$ are measurable and distinct,

(4) (Martin, Kunen 1971) $\boldsymbol{\delta}_{2}^{1}=\aleph_{2}, \boldsymbol{\delta}_{3}^{1}=\aleph_{\omega+1}$, and $\boldsymbol{\delta}_{4}^{1}=\aleph_{\omega+2}$,

(5) (Martin, Paris 1971) $\boldsymbol{\delta}_{1}^{1} \rightarrow\left(\boldsymbol{\delta}_{1}^{1}\right)^{1}$, and for all $\alpha<\boldsymbol{\delta}_{2}^{1}$, the relation $\boldsymbol{\delta}_{2}^{1} \rightarrow$ $\left(\boldsymbol{\delta}_{2}^{1}\right)^{\alpha}$ holds, 
(6) (Martin 1971) for all $\alpha<\omega_{1}$ the partition relation $\boldsymbol{\delta}_{2 n+1}^{1} \rightarrow\left(\boldsymbol{\delta}_{2 n+1}^{1}\right)^{\alpha}$ holds,

(7) (Kunen 1971) the $\omega$-cofinal measure $\mathcal{C}_{\boldsymbol{\delta}_{2 n+1}^{1}}^{\omega}$ is a normal measure on $\boldsymbol{\delta}_{2 n+1}^{1}$ with $\boldsymbol{\delta}_{2 n+1}^{1} \boldsymbol{\delta}_{2 n+1}^{1} / \mathcal{C}_{\boldsymbol{\delta}_{2 n+1}^{1}}^{\omega}=\boldsymbol{\delta}_{2 n+2}^{1}=\left(\boldsymbol{\delta}_{2 n+1}^{1}\right)^{+}$, and

(8) (Martin, Jackson 1980) $\boldsymbol{\delta}_{3}^{1^{\delta_{3}^{1}}} / \mathcal{C}_{\boldsymbol{\delta}_{3}^{1}}^{\omega_{1}}=\aleph_{\omega \cdot 2+1}=\left(\boldsymbol{\delta}_{3}^{1}\right)^{(\omega+1)}$ and $\boldsymbol{\delta}_{3}^{1^{\delta_{3}^{1}}} / \mathcal{C}_{\boldsymbol{\delta}_{3}^{1}}^{\omega_{2}}=$ $\aleph_{\omega^{\omega}+1}=\left(\boldsymbol{\delta}_{3}^{1}\right)^{\left(\omega^{\omega}+1\right)}$, and these two cardinals are measurable.

Proof. A proof of all parts except for the last can be found in [Ke78]. Fact 5 comprises of Theorem 3.12, Theorem 3.10, Theorem 5.1, §6, Theorem 12.1, Corollary 13.4, Theorem 11.2, and Theorem 14.3 of [Ke78]. The last is part of [Ja99, Chapter 7].

Theorem 5 gives an indication of how representing cardinals as ultrapowers of measures helps in computations. Let us make this more explicit in one example: Suppose we are working in AD, we know that $\boldsymbol{\delta}_{2}^{1}=\aleph_{2}$ and we know that for each $n>2$ there is some measure $\mu$ on $\aleph_{1}$ such that $\delta_{2}^{1} \omega_{1} / \mu=\aleph_{n}$. Suppose furthermore that it is our goal to compute $\boldsymbol{\delta}_{3}^{1}$ (so, we are trying to prove part of Theorem 5 (4)). Tony Martin showed that $\boldsymbol{\delta}_{3}^{1} \leq \aleph_{\omega+1}$ (just from the existence of sharps; $c f$. [Ka94, p. 428]); by Theorem 5 (3), we know that $\boldsymbol{\delta}_{3}^{1}>\boldsymbol{\delta}_{2}^{1}=\aleph_{2}$ is a regular cardinal, so there are only two options left: either $\boldsymbol{\delta}_{3}^{1}=\aleph_{n}$ for some $n>2$ or $\boldsymbol{\delta}_{3}^{1}=\aleph_{\omega+1}$.

We can finish our computation of $\boldsymbol{\delta}_{3}^{1}$ with the following lemma which will be of use later in the proof of our main theorem:

Lemma 6 . Let $\kappa<\lambda$ be cardinals, $\mu$ a measure on $\kappa$ and $\operatorname{cf}(\lambda)>\kappa$. Then $\operatorname{cf}\left(\lambda^{\kappa} / \mu\right)=\operatorname{cf}(\lambda)$.

Proof. " $\leq$ ": For $\alpha<\lambda$, let $c_{\alpha}: \kappa \rightarrow \lambda$ be the constant function $c_{\alpha}(\xi)=\alpha$. We shall show that $\left\{\left[c_{\alpha}\right]_{\mu} ; \alpha \in \lambda\right\}$ is cofinal in $\lambda^{\kappa} / \mu$ :

Let $f \in \lambda^{\kappa}$ be arbitrary. Since $\operatorname{cf}(\lambda)>\kappa$, the range of the function $f$ is bounded in $\lambda$, i.e., there is an $\alpha^{*} \in \lambda$ such that $\{f(\xi) ; \xi \in \kappa\} \subseteq \alpha^{*}$. Then $[f]_{\mu}<\left[c_{\alpha^{*}}\right]_{\mu}$.

" $\geq$ ": Now let $X \subseteq \lambda^{\kappa} / \mu$ be a cofinal subset. If $\xi \in X$, there is some $\alpha \in \lambda$ such that $\xi \leq\left[c_{\alpha}\right]_{\mu}$ by the above argument. Let $\alpha_{\xi}$ be the least such ordinal. We claim that $A:=\left\{\alpha_{\xi} ; \xi \in X\right\}$ is a cofinal subset of $\lambda$ : Let $\gamma \in \lambda$ be arbitrary. Since $X$ was cofinal, pick some $\xi_{\gamma} \in X$ such that $\xi_{\gamma}>\left[c_{\gamma}\right]_{\mu}$. But then, $\alpha_{\xi_{\gamma}} \in A$ with $\alpha_{\xi_{\gamma}}>\gamma$. So, $A$ is cofinal in $\lambda$. But $\operatorname{Card}(A) \leq \operatorname{Card}(X)$, so $\operatorname{cf}(\lambda) \leq \operatorname{cf}\left(\lambda^{\kappa} / \mu\right)$.

As a consequence of Lemma 6 , we get from our assumptions that $\operatorname{cf}\left(\aleph_{n}\right)=\aleph_{2}$ for $n>2$, and thus these cardinals are singular. This leaves $\boldsymbol{\delta}_{3}^{1}=\aleph_{\omega+1}$ as the last remaining possibility and the computation of $\boldsymbol{\delta}_{3}^{1}$ is finished.

By carrying information about the cofinality of the cardinals, the representation of the $\aleph_{n}$ as ultrapowers allowed us to exclude them from the list of candidates for being $\boldsymbol{\delta}_{3}^{1}$. This will be a recurring idea for the main proof in this paper. This little argument is paradigmatic for measure analyses; ideas like this were fully exploited in the work of Steve Jackson when he computed all of the projective ordinals under the assumption of $A D$.

Theorem 7. Assume AD. Let $\mathbf{e}_{0}:=0$ and $\mathbf{e}_{n+1}:=\omega^{\left(\omega^{\mathbf{e}_{n}}\right)}$ (i.e., $\mathbf{e}_{n}$ is a exponential $\omega$-tower of height $2 n-1)$. Then for every $n \in \omega$,

$$
\boldsymbol{\delta}_{2 n+1}^{1}=\aleph_{\mathbf{e}_{n}+1},
$$


and all odd projective ordinals have the strong partition property.

Proof. The original paper is [Ja88]; a more accessible (but still very involved) proof of the case $n=2$ can be found in [Ja99].

The proof of Theorem 7 is outside of the scope of this paper, but deeply connected with the techniques we are using. Jackson's proof gives more than just a computation of the projective ordinals. Along the way, Jackson gives an analysis of many (in a sense, almost all) cardinals $\kappa<\aleph_{\varepsilon_{0}}=\sup \left\{\boldsymbol{\delta}_{n}^{1} ; n \in \omega\right\}$ in terms of ultrapowers of the projective ordinals.

1.3. Measure Analyses. By the term "measure analysis", we shall understand informally the following procedure: given a strong partition cardinal $\kappa$ and some cardinal $\lambda>\kappa$, we assign a measure $\mu$ on $\kappa$ to $\lambda$ such that $\kappa^{\kappa} / \mu=\lambda$. A central tool for measure analyses is the following theorem of Martin's on measures on strong partition cardinals:

Theorem 8 (Martin). Let $\kappa$ be a strong partition cardinal and let $\mu$ be a measure on $\kappa$. Then the ultrapower $\kappa^{\kappa} / \mu$ is a cardinal.

Proof. [Ja99, Theorem 7.1].

Of course, a measure analysis can only be fruitfully applied if it is a uniform assignment of measures to cardinals. In order to provide such an assignment, we shall use the notions of ordinal algebras and canonical measure assignments that were developed by Jackson and the second author in the fall of 2003 (more details will be contained in the paper [JaLö $\infty])$.

An ordinal algebra is an algebra $\mathfrak{A}$ with binary operations $\oplus$ and $\otimes$ on a set of generators $\mathfrak{V}$ together with an epimorphism

$$
o: \text { Free }_{\oplus, \otimes}(\mathfrak{V}) \rightarrow \alpha
$$

where $\alpha$ is an ordinal such that the relations of $\mathfrak{A}$ are given by $x=y$ for all $x, y$ such that $o(x)=o(y)$. We introduce a notation for finitely iterated sums and products:

$$
\begin{aligned}
\mathrm{V} \otimes n & :=\mathrm{V} \underbrace{\oplus \ldots \oplus}_{n} \mathrm{~V}, \text { and } \\
\mathrm{V}^{\otimes n}: & :=\mathrm{V} \underbrace{\otimes \ldots \otimes}_{n} \mathrm{~V} .
\end{aligned}
$$

The ordinal $\alpha$ is called the height of $\mathfrak{A}$, in symbols ht( $\mathfrak{A})$. An ordinal algebra $\mathfrak{A}$ is little more than a formal syntactic way of describing the ordinal ht( $\mathfrak{A})$. It is uniquely determined by the order type of its set of generators. We write $\mathfrak{A}_{\alpha}$ for the ordinal algebra with generators of order type $\alpha$. Let us look at the simplest ordinal algebras as an example:

- $(\alpha=1)$. If $\mathfrak{V}=\left\{\mathrm{V}_{0}\right\}$, then $o\left(\mathrm{~V}_{0}\right)=0$, so $\mathrm{V}_{0} \oplus \mathrm{V}_{0}=\mathrm{V}_{0}$ and $\mathrm{V}_{0} \otimes \mathrm{V}_{0}=\mathrm{V}_{0}$, so $\mathfrak{A}$ has one element, and $h t(\mathfrak{A})=1$.

- $(\alpha=2)$. If $\mathfrak{V}=\left\{\mathrm{V}_{0}, \mathrm{~V}_{1}\right\}$, then $o\left(\mathrm{~V}_{0}\right)=0$ and $o\left(\mathrm{~V}_{1}\right)=1$, so $\mathrm{V}_{0} \oplus \mathrm{V}_{0}=\mathrm{V}_{0}$, $\mathrm{V}_{0} \otimes \mathrm{V}_{0}=\mathrm{V}_{0}, \mathrm{~V}_{0} \otimes \mathrm{V}_{1}=\mathrm{V}_{0}, \mathrm{~V}_{1} \otimes \mathrm{V}_{1}=\mathrm{V}_{1}$, so ht $(\mathfrak{A})=\omega$.

Proposition 9 .

$$
\operatorname{ht}\left(\mathfrak{A}_{\alpha}\right):=\left\{\begin{array}{cl}
1 & \alpha=1 \\
\omega^{\omega^{n-2}} & 1<\alpha=n<\omega \\
\omega^{\omega^{\alpha}} & \alpha \geq \omega .
\end{array}\right.
$$


Proof. We'll prove the claim by induction on $\alpha$. Suppose that $h t\left(\mathfrak{A}_{\alpha}\right)=\omega^{\omega^{\alpha}}$. Let us add a new generator $\mathrm{V}$ to $\mathfrak{A}_{\alpha}$ in order to construct $\mathfrak{A}_{\alpha+1}$. Note that $o(\mathrm{~V})$ must be $\operatorname{ht}\left(\mathfrak{A}_{\alpha}\right)=\omega^{\omega^{\alpha}}$. Since $o$ is an epimorphism, we get that

$$
\begin{gathered}
o\left(\mathrm{~V}^{\otimes n}\right)=\left(\omega^{\omega^{\alpha}}\right)^{n}=\omega^{\left(\omega^{\alpha}\right) \cdot n}, \text { so } \\
\operatorname{ht}\left(\mathfrak{A}_{\alpha+1}\right)=\sup \left\{\omega^{\left(\omega^{\alpha}\right) \cdot n} ; n \in \omega\right\}=\omega^{\left(\omega^{\alpha}\right) \cdot \omega}=\omega^{\omega^{\alpha+1}} .
\end{gathered}
$$

As a side remark that cannot be fully explored in this paper, we should like to mention that Proposition 9 is the ordinal-theoretic reflection of Jackson's computation of the projective ordinals: if $\boldsymbol{\delta}_{2 n-1}^{1}=\aleph_{\alpha+1}$, then $\boldsymbol{\delta}_{2 n+1}^{1}=\aleph_{\omega^{\omega^{\alpha}+1}}$ since the cardinal structure between $\boldsymbol{\delta}_{2 n-1}^{1}$ and $\boldsymbol{\delta}_{2 n+1}^{1}$ is determined by the ordinal algebra $\mathfrak{A}_{\alpha}$.

1.4. Algebras of measures and measure assignments. Given a strong partition cardinal $\kappa$ and an ordinal algebra $\langle\mathfrak{A}, o\rangle$, we shall now sketch how we assign measures on $\kappa$ to the elements of the ordinal algebra. We want to assign measures to the generators and then homomorphically extend the assignment to all elements of the ordinal algebra. The usual notions of sum and product of measures are not appropriate for this, as they tend to give equivalent measures. We therefore need to capitalize on the strong partition property of $\kappa$ and the fact that this gives us an algebraic structure on a particular class of measures, the order measures. We have to prepare the definition of order measures and the operations on them with some preliminary definitions:

Let $\kappa$ and $\alpha$ be ordinals. A function $f: \alpha \rightarrow \kappa$ is continuous ${ }^{[4]}$ if and only if for all limit ordinals $\lambda<\alpha$,

$$
f(\lambda)=\sup \{f(\xi) ; \xi<\lambda\} .
$$

The function $f$ has uniform cofinality $\omega$ if there is a function $h: \omega \times \alpha \rightarrow \kappa$, which is increasing in the first argument, such that for $\gamma<\alpha$, we have (for $k<\omega$ and $\gamma \geq \omega)$

$$
\begin{aligned}
f(k) & =\sup \{h(n, k) ; n \in \mathbb{N}\}, \text { and } \\
f(\gamma+1) & =\sup \{h(n, \gamma) ; n \in \mathbb{N}\} .
\end{aligned}
$$

We say that a function $f: \alpha \rightarrow \kappa$ has type $\alpha$ if it is increasing, continuous and has uniform cofinality $\omega$. If $X \subseteq \kappa$, we write $\mathcal{F}_{\alpha}^{X}$ for the set of functions from $\alpha$ to $X$ that are of type $\alpha$. We write $\mathcal{F}_{\alpha}:=\mathcal{F}_{\alpha}^{\kappa}$. If $C \subseteq \kappa$ is club and and $\alpha$ is an element of $\kappa$, we write $C_{\alpha}$ for the club set of elements in $C$ that are greater than $\alpha$, i.e., $C_{\alpha}:=\{\beta \in C ; \beta>\alpha\}$.

Now let $\left\langle\varrho_{i} ; i<n\right\rangle$ be a sequence of successor ordinals less than $\kappa$ and $\left\langle\mu_{i} ; i<\right.$ $n\rangle$ a sequence of measures such that $\mu_{i}$ is a measure on $\varrho_{i}$ and $\kappa^{\varrho_{i}} / \mu_{i}=\kappa$ for all $i<n$. Let $\varrho:=\sum_{i<n} \varrho_{i}$. For any function $f: \varrho \rightarrow \kappa$, we can define (for each $i<n$ )

$$
f_{i}(\alpha):=f\left(\sum_{j<i} \varrho_{j}+\alpha\right),
$$

\footnotetext{
${ }^{[4]}$ For increasing functions, this is the ordinary notion of continuity for the order topology on ordinals.
} 
and get a sequence $\vec{f}:=\left\langle f_{i} ; i<n\right\rangle$ of functions $f_{i}: \varrho_{i} \rightarrow \kappa$. If the original $f$ was of type $\varrho$, then $f_{i} \in \mathcal{F}_{\varrho_{i}}$ and $f_{i+1}(0)>\sup f_{i}$. We define ${ }^{[5]}$

$$
\begin{aligned}
& \operatorname{lift}(\vec{\mu}, \vec{\varrho}):=\left\{A \subseteq \kappa \quad ; \quad \text { there is a club set } C \subseteq \kappa \text { such that for all } f \in \mathcal{F}_{\varrho}^{C}\right. \\
& \text { we have } \left.\left\ulcorner\left[f_{0}\right]_{\mu_{0}}, \ldots,\left[f_{n-1}\right]_{\mu_{n-1}}\right\urcorner \in A\right\} \\
& =\{A \subseteq \kappa ; \text { there is a club set } C \subseteq \kappa \text { such that } \\
& \text { for all } x_{0} \in \mathcal{F}_{\varrho_{0}}^{C}, x_{1} \in \mathcal{F}_{\varrho_{1}}^{C_{\text {sup } x_{0}}}, \ldots, x_{n-1} \in \mathcal{F}_{\varrho_{n-1}}^{C_{\text {sup } x_{n-2}}} \\
& \text { we have } \left.\left\ulcorner\left[x_{0}\right]_{\mu_{0}}, \ldots,\left[x_{n-1}\right]_{\mu_{n-1}}\right\urcorner \in A\right\} \text {. }
\end{aligned}
$$

TheOREM 10 (Lifting Theorem). Let $\kappa$ be a strong partition cardinal and $n \in \omega$. Let $\vec{\varrho} \in \kappa^{n}$ be a sequence of successor ordinals and $\vec{\mu}$ a sequence of measures such that $\mu_{i}$ is a measure on $\varrho_{i}$ and $\kappa^{\varrho_{i}} / \mu_{i}=\kappa$ for all $i<n$. Then $\operatorname{lift}(\vec{\mu}, \vec{\varrho})$ is a measure on $\kappa$.

Proof. Using Lemma 4, we can choose from countable families of nonempty sets of subsets of $\kappa$, so the club filter on $\kappa$ is $\sigma$-complete and thus $\operatorname{lift}(\vec{\mu}, \vec{\varrho})$ is a $\sigma$-complete filter. We have to show the "ultra" property:

Let $A \subseteq \kappa$ be fixed and let $\varrho:=\sum_{i<n} \varrho_{i}$. We define a colouring of $\kappa^{\varrho}$ as follows:

$$
c_{A, \vec{\mu}}(f):= \begin{cases}1 & \text { if }\left\ulcorner\left[f_{0}\right]_{\mu_{0}}, \ldots,\left[f_{n-1}\right]_{\mu_{n-1}}\right\urcorner \in A, \\ 0 & \text { otherwise. }\end{cases}
$$

If we can show that there is a club set $C$ such that $\mathcal{F}_{\varrho}^{C}$ is homogeneous for $c_{A, \vec{\mu}}$, then we're done (since then either $A \in \operatorname{lift}(\vec{\mu}, \vec{\varrho})$ or $\kappa \backslash A \in \operatorname{lift}(\vec{\mu}, \vec{\varrho})$ ).

For an element $g \in \kappa^{\omega \times \varrho}, k<\omega, \gamma \geq \omega$ and $\lambda$ a limit ordinal, we define

$$
\begin{aligned}
\hat{g}(k) & :=\sup \{g(n, k) ; n \in \mathbb{N}\}, \\
\hat{g}(\gamma+1) & :=\sup \{g(n, \gamma) ; n \in \mathbb{N}\}, \text { and } \\
\hat{g}(\lambda) & :=\sup \{\hat{g}(\eta) ; \eta<\lambda\} .
\end{aligned}
$$

and define an auxiliary colouring of $\kappa^{\omega \times \varrho}$ by

$$
G(g):=c_{A, \vec{\mu}}(\hat{g})
$$

Since $\kappa \rightarrow(\kappa)^{\kappa}$ holds, there is a homogeneous set $H$ of size $\kappa$ for $G$. Let $C$ be the set of limit points in $H$. Clearly, $C$ is a club subset of $\kappa$, so we shall be finished if we show that $\mathcal{F}_{\varrho}^{C}$ is homogeneous for $c_{A, \vec{\mu}}$. We shall show that for each $f \in \mathcal{F}_{\varrho}^{C}$, there is some $g \in H^{\omega \times \varrho}$ such that $c_{A, \vec{\mu}}(f)=G(g)$.

Let $f \in \mathcal{F}_{\varrho}^{C}$. By assumption ( $f$ has uniform cofinality $\omega$ ), there is some $h$ : $\omega \times \varrho \rightarrow \kappa$ such that for all $k<\omega$ and $\omega \leq \gamma<\varrho$, we have

$$
\begin{aligned}
f(k) & :=\sup \{h(n, k) ; n \in \mathbb{N}\} \\
f(\gamma+1) & =\sup \{h(n, \gamma) ; n \in \mathbb{N}\}
\end{aligned}
$$

in other words, $\hat{h}=f$. We define

$$
g(n, \gamma):=\min \{\delta \in H ; \delta \geq h(n, \gamma)\}
$$

Since $f(\gamma+1)=\sup \{h(n, \gamma) ; n \in \mathbb{N}\} \in C$, we get that $\hat{g}=\hat{h}=f$.

${ }^{[5]}$ Here and for the rest of the paper, $\left.\ulcorner.\urcorner,\right\urcorner$ is the Gödel pairing function, i.e., a definable bijection between $\kappa \times \kappa$ and $\kappa$. Similarly, $\ulcorner\urcorner:. \kappa^{n} \rightarrow \kappa$ is a definable bijection between $\kappa^{n}$ and $\kappa$. 
With Theorem 10 we can now define the most important notion for measure analyses under AD: If $\mu$ is a measure on $\kappa$, we say that $\mu$ is an order measure if $\mu=\operatorname{lift}(\vec{\nu}, \vec{\varrho})$ for some sequence of successor ordinals $\vec{\varrho} \in \kappa^{n}$ and $\vec{\nu}$ a sequence of measures such that $\nu_{i}$ is a measure on $\varrho_{i}$ and $\kappa^{\varrho_{i}} / \nu_{i}=\kappa$ for all $i<n$. In this case, we call germ ger $_{\mu}:=\vec{\nu}$ the germ of $\mu$ and o.t. $\mu:=\vec{\varrho}$ the order type of $\mu$. To denote the elements of the germ and the order type we write $\operatorname{germ}_{\mu, i}:=\nu_{i}$ and o.t. $\mu, i=\varrho_{i}$. We let $\ln _{\mu}:=\ln \left(\right.$ germ $\left._{\mu}\right)-1=n-1$.

OBservation 11. The $\omega$-cofinal measure $\mathcal{C}_{\kappa}^{\omega}$ is an order measure since $\mathcal{C}_{\kappa}^{\omega}=$ $\operatorname{lift}\left(\mu_{\{\omega\}}, \omega+1\right)$ where $\mu_{\{\omega\}}$ is the principal measure that concentrates on $\omega$. Similarly, $\mathcal{C}_{\kappa}^{\omega_{1}}=\operatorname{lift}\left(\mathcal{C}_{\omega_{1}}^{\omega}, \omega_{1}+1\right)$. (clearly, if $\mu$ is a measure on $\kappa$, then we can see it trivially as a measure on $\kappa+1$ by giving the additional point measure 0 ).

We can now define a binary operation on order measures: Let $\mu$ and $\nu$ be order measures with $\mu=\operatorname{lift}\left(\operatorname{germ}_{\mu}\right.$, o.t. $\left.\mu\right)$ and $\nu=\operatorname{lift}\left(\operatorname{germ}_{\nu}\right.$, o.t. $\left._{\cdot}\right)$. Then the sum of the order measures $\mu$ and $\nu$, written $\mu \oplus \nu$, is the order measure $\operatorname{lift}\left(\operatorname{germ}_{\mu_{\oplus} \nu}\right.$, o.t. $\left.\mu \oplus \nu\right)$ with

$$
\operatorname{germ}_{\mu_{\oplus} \nu}:=\operatorname{germ}_{\mu} \frown \operatorname{germ}_{\nu}
$$

and

$$
\text { o.t. } \mu_{\oplus} \nu:=\text { o.t. } \mu^{\frown \text { o.t. }} \cdot \nu \text {. }
$$

Let us spell out what this means concretely. For this, we will write $\sup \vec{x}:=$ $\sup \left\{\sup x_{i} ; i<\operatorname{lh}(\vec{x})\right\}$, use the abbreviation " $\vec{x} \in \mathcal{F}_{\text {o.t. } \mu}^{C}$ " for the statement "for all $i \leq \ln _{\mu}$, we have $x_{i} \in \mathcal{F}_{\text {o.t. } ., i^{\sup _{i-1}}}^{C_{1},[6]}$ and write $\overrightarrow{[x]}_{\text {germ }_{\mu}}$ for the sequence $\left\langle\left[x_{0}\right]_{\operatorname{germ}_{\mu, 0}}, \ldots,\left[x_{\ln _{\mu}}\right]_{\left.\operatorname{germ}_{\mu, \mathbf{l n}_{\mu}}\right\rangle:}\right.$

$$
\begin{aligned}
& A \in \mu \oplus \nu \quad \Leftrightarrow \quad \text { there is a club set } C \subseteq \kappa \text { such that } \\
& \text { for all } \vec{x} \in \mathcal{F}_{\text {o.t. } \mu}^{C}, \vec{y} \in \mathcal{F}_{\text {o.t. }{ }_{\nu}}^{C_{\text {sup }}} \text { we have }
\end{aligned}
$$

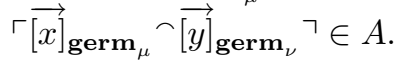

We can also define a binary operation $\otimes$ that is based on taking products of germs and order types in the same way that $\oplus$ is based on sums. Since $\otimes$ will play no rôle in this paper, we shall not discuss it in detail. With the operations $\oplus$ and $\otimes$, we can now come back to the notion of a "measure assignment":

A measure assignment for $\kappa$ and $\mathfrak{A}$ is a function meas assigning an order measure on $\kappa$ to each generator of $\mathfrak{A}$. If the function meas is well-behaved, we can extend the function meas to a function on $\mathfrak{A}$ with the binary operations $\oplus$ and $\otimes$. ${ }^{[7]}$ We now call a measure assignment canonical if for all $x \in \mathfrak{A}$ with $o(x)=1+\alpha$, we have

$$
\kappa^{\kappa} / \operatorname{meas}(x)=\kappa^{(\alpha+1)},
$$

and the ultrapower does not depend on the presentation of $x$ (in the sense of Footnote [7]).

\footnotetext{
${ }^{[6]}$ Here, $\sup x_{-1}:=-1$.

$\left.{ }^{[7}\right]_{E . g .,}$ if $o\left(\mathrm{~V}_{1}\right)=1, o\left(\mathrm{~V}_{2}\right)=\omega, \operatorname{meas}\left(\mathrm{V}_{1}\right)=\mu$ and meas $\left(\mathrm{V}_{2}\right)=\nu$, then $\nu$ and $\mu \oplus \nu$ should not be radically different (as they correspond to the same ordinal $\omega=1+\omega$ ).
} 
Rephrased in this language, we can say that Kleinberg's Theorem 3 gives a canonical measure assignment for $\aleph_{1}$ and $\mathfrak{A}_{2}=\mathfrak{A}_{2+\mathbf{e}_{0}} \cdot{ }^{[8]}$ The mentioned analysis by Jackson and Khafizov can now be phrased as follows:

THEOREM 12 (Jackson-Khafizov). There is a canonical measure assignment for $\boldsymbol{\delta}_{3}^{1}=\boldsymbol{\delta}_{2 \cdot 1+1}^{1}$ and $\mathfrak{A}_{\omega}=\mathfrak{A}_{\mathbf{e}_{1}}$.

In general, we expect that there is a canonical measure assignment for $\boldsymbol{\delta}_{2 n+1}^{1}$ and $\mathfrak{A}_{\mathbf{e}_{n}}$. The main result of this paper is a very modest first step towards such a result. Let us look at $\mathfrak{A}_{3}$. If we restrict $\mathfrak{A}_{3}$ to the operation $\oplus$, we get an ordinal algebra $\mathfrak{A}_{3}^{\oplus}$ of height $\omega^{2}$ that we shall call the $\oplus$-reduct of $\mathfrak{A}_{3}$. We shall be able to show that for all $n>0$ there is a canonical measure assignment for $\boldsymbol{\delta}_{2 n+1}^{1}$ and $\mathfrak{A}_{3}^{\oplus}$ (Corollary 24).

1.5. Applications. If we have a canonical measure assignment and some information about the measures assigned to the generators, then we can use this in order to get information about the cardinals being represented. As mentioned, Kleinberg's Theorem 3 gives us partition properties for cardinals that can be represented as iterated ultrapowers of normal measures. If we can connect the iterated ultrapowers of normal measures to the ultrapowers with measures occurring in the algebra of measures, then we can compute the Kleinberg sequences. In general, we can embed the sum of two measures into the iterated ultrapower: ${ }^{[9]}$

Proposition 13. Let $\kappa$ be a strong partition cardinal and let $\mu$ and $\nu$ be order measures, both on $\kappa$. Let $\lambda \geq \kappa$ be a cardinal. Then

$$
\lambda^{\kappa} /(\mu \oplus \nu) \leq\left(\lambda^{\kappa} / \nu\right)^{\kappa} / \mu \text {. }
$$

Proof. For $f: \kappa \rightarrow \lambda$ define $\hat{f}: \kappa \rightarrow \lambda^{\kappa} / \nu$ by $\left.\hat{f}(\ulcorner\vec{\alpha}\urcorner):=[\ulcorner\vec{\beta}\urcorner \mapsto f(\ulcorner\vec{\alpha}\urcorner \vec{\beta}\urcorner)\right]_{\nu}$. We shall show that $f \rightarrow \hat{f}$ induces an embedding from $\lambda^{\kappa} /(\mu \oplus \nu)$ into $\left(\lambda^{\kappa} / \nu\right)^{\kappa} / \mu$. ${ }^{[10]}$ If $[f]_{\mu \oplus \nu} \leq[g]_{\mu \oplus \nu}$, then there is a club set $C \subseteq \kappa$ such that for all $\vec{x} \in \mathcal{F}_{\text {o.t. } \mu}^{C}$ and all $\vec{y} \in \mathcal{F}_{{\text {o.t. }{ }_{\nu}}^{C_{\text {sup }}}}$ we have

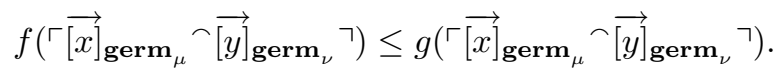

Momentarily fixing $\vec{x}$ in this statement and observing that $C_{\sup } \vec{x}$ is club in $\kappa$, we get that there is a club set $C \subseteq \kappa$ such that for all $\vec{x} \in \mathcal{F}_{\text {o.t. }{ }_{\mu}}^{C}$ we have

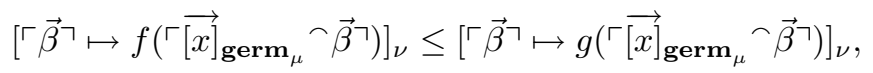

which by definition of $f \mapsto \hat{f}$ translates to $[\hat{f}]_{\mu} \leq[\hat{g}]_{\mu}$.

\footnotetext{
${ }^{[8]}$ Of course, Kleinberg's ultrapower representations of the $\aleph_{n}$ are not of the form $\kappa^{\kappa} / \mu$ for a measure $\mu ; c f$. Corollary 17 .

${ }^{[9]}$ It is not a coincidence that our measure assignment in Corollary 24 only works for the $\oplus$-reduct. In order to extend our argument to full ordinal algebras, we should need an analogue of Proposition 13 for the operation $\otimes$, i.e., an operation that corresponds to $\otimes$ in the same sense as iterated ultrapowers correspond to $\oplus$.

${ }^{[10]}$ In order to show that $f \mapsto \hat{f}$ induces an embedding, we have to show two properties:

(1) the function is welldefined, i.e., if $[f]_{\mu \oplus \nu}=[g]_{\mu \oplus \nu}$, then $[\hat{f}]_{\mu}=[\hat{g}]_{\mu}$, and

(2) the function induces an injection, i.e., if $[f]_{\mu \oplus \nu}<[g]_{\mu \oplus \nu}$, then $[\hat{f}]_{\mu}<[\hat{g}]_{\mu}$.

Obviously, the proofs of these two statements are typically parallel, and we reduce them in most places of this paper to one proof where we show the implication for $\leq$. This is supposed to be understood properly as " $</="$.
} 
The identification of iterated ultrapowers (as they occur in Kleinberg sequences) and ultrapowers with order measures is the methodology behind Kleinberg's original computation and its generalizations to $\boldsymbol{\delta}_{2 n+1}^{1}$ (using Theorem $5(7)$ ). This will be discussed in Corollary 17, after we provided the last missing piece in the inductive mosaic.

\section{Embeddings between ultrapowers of order measures}

In this section, we'll give the necessary embedding results for order measures needed in our inductive proof of the main theorem.

Lemma 14. Let $\kappa$ be a strong partition cardinal and let $\mu$ and $\nu$ be order measures on $\kappa$. Then

(1) $\kappa^{\kappa} / \mu \leq \kappa^{\kappa} /(\mu \oplus \nu)$, and

(2) $\kappa^{\kappa} / \nu \leq \kappa^{\kappa} /(\mu \oplus \nu)$.

Proof. For $f: \kappa \rightarrow \kappa$, we define $f_{0}, f_{1}: \kappa \rightarrow \kappa$ by $\left.f_{0}(\ulcorner\vec{\alpha}\urcorner \vec{\beta}\urcorner\right):=f(\ulcorner\vec{\alpha}\urcorner)$ and $f_{1}(\ulcorner\vec{\alpha} \neg \vec{\beta}\urcorner):=f(\ulcorner\vec{\beta}\urcorner)$. Now $f \rightarrow f_{0}$ induces an embedding from $\kappa^{\kappa} / \mu$ into $\kappa^{\kappa} /(\mu \oplus \nu)$ and $f \rightarrow f_{1}$ induces an embedding from $\kappa^{\kappa} / \nu$ into $\kappa^{\kappa} /(\mu \oplus \nu)$. As the proofs are identical, let us just look at (2):

$$
\begin{aligned}
& {[f]_{\nu} \leq[g]_{\nu} \Leftrightarrow \text { there is a club set } C \subseteq \kappa \text { such that }} \\
& \text { for all } \left.\left.\vec{y} \in \mathcal{F}_{\text {o.t. }}^{C} \text {, we have } f\left(\ulcorner\overrightarrow{[y}]_{\text {germ }_{\nu}}\right\urcorner\right) \leq g\left(\ulcorner\overrightarrow{[y]}]_{\text {germ }_{\nu}}\right\urcorner\right) \text {. } \\
& \Rightarrow \text { there is a club set } C \subseteq \kappa \text { such that } \\
& \text { for all } \vec{x} \in \mathcal{F}_{\text {o.t. }}^{C}, \vec{y} \in \mathcal{F}_{\text {o.t. }{ }^{\prime}}^{C_{\text {sup }}} \text { we have }
\end{aligned}
$$

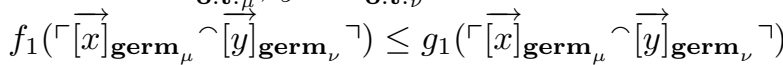

$$
\begin{aligned}
& \Leftrightarrow\left[f_{1}\right]_{\mu \oplus \nu} \leq\left[g_{1}\right]_{\mu \oplus \nu} .
\end{aligned}
$$

Lemma 15. Let $\lambda<\kappa$ be regular cardinals and $\gamma<\lambda$. Let $C$ be a given club set in $\kappa$ and $\Gamma:[\kappa]^{\gamma} \rightarrow \kappa$. Then there is a club set $C^{*} \subseteq C$ such that for all $\alpha \in C^{*}$ and $x \in[\alpha]^{\gamma}$, we have $\Gamma(x)<\alpha$.

Proof. Let $C^{*}:=\left\{\alpha \in C ; \operatorname{cf}(\alpha) \geq \lambda\right.$ and for all $x \in[\alpha]^{\gamma}$ we have $\left.\Gamma(x)<\alpha\right\}$. Obviously, $C^{*}$ is closed. We'll show that it is unbounded in $\kappa$ : fix some $\xi \in \kappa$. For $\eta<\lambda$, let us define recursively $\xi_{\eta+1}:=\max \left\{\xi_{\eta}+1, \sup \left\{\Gamma(x) ; x \in\left[\xi_{\eta}\right]^{\gamma}\right\}\right\}$ and (if $\eta$ is a limit) $\xi_{\eta}:=\sup \left\{\xi_{\zeta} ; \zeta<\eta\right\}$. Let $\alpha:=\sup \left\{\xi_{\eta} ; \eta<\lambda\right\}$, so $\operatorname{cf}(\alpha)=\lambda$ (as the sequence is strictly increasing). Since $\lambda$ is regular and $\gamma<\lambda$, we have that $\alpha \in C^{*}$.

Proposition 16. Let $\lambda<\kappa$ be regular cardinals with $\kappa \rightarrow(\kappa)^{\kappa}$. Let $\mu$ be an order measure on $\kappa$ with o.t. $\mu_{, i}<\lambda$ for all $i \leq \ln _{\mu}$. Then

$$
\left(\kappa^{\kappa} / \mu\right)^{+} \leq \kappa^{\kappa} /\left(\mu \oplus \mathcal{C}_{\kappa}^{\omega}\right) \text {. }
$$

Proof. For $f: \kappa \rightarrow \kappa$ define $\hat{f}: \kappa \rightarrow \kappa$ by $\hat{f}\left(\left\ulcorner\vec{\alpha}^{\wedge} \beta\right\urcorner\right):=f(\ulcorner\vec{\alpha}\urcorner)$. By Lemma 14 this induces an embedding from $\kappa^{\kappa} / \mu$ into $\kappa^{\kappa} /\left(\mu \oplus \mathcal{C}_{\kappa}^{\omega}\right)$. We'll show that this embeds $\kappa^{\kappa} / \mu$ into a proper initial segment of $\kappa^{\kappa} /\left(\mu \oplus \mathcal{C}_{\kappa}^{\omega}\right)$ which is enough by Martin's Theorem 8. Let $f \in \kappa^{\kappa}$ be arbitrary and let $\pi$ be defined by $\pi\left(\left\ulcorner\vec{\alpha}^{\wedge} \beta\right\urcorner\right):=\beta$. We shall show that

$$
[\hat{f}]_{\mu \oplus \mathcal{C}_{\kappa}^{\omega}}<[\pi]_{\mu \oplus \mathcal{C}_{\kappa}^{\omega}}
$$


By applying Lemma 15 several times, we can get a club set $C$ such that for all $\alpha \in C$, we have

(1) if $\beta<\alpha$, we have $f(\beta)<\alpha$,

(2) for all $i \leq \ln _{\mu}$, if $x_{i} \in[\alpha]^{\text {o.t. } \mu, i}$, we have $\left[x_{i}\right]_{\mathbf{g e r m}_{\mu, i}}<\alpha$, and

(3) if $\vec{\beta} \in[\alpha]^{\ln \mu}$, we have $\ulcorner\vec{\beta}\urcorner<\alpha$.

Now take $\vec{x} \in \mathcal{F}_{\text {o.t. } \mu^{C}}$ and $y \in \mathcal{F}_{\omega+1}^{C_{\text {sup } \vec{x}}}$ arbitrary. Since $y(\omega) \in C$ and $x_{i} \in[y(\omega)]^{\text {o.t. }{ }_{\text {. }, i}}$ for all $i \leq \ln _{\mu}$, by property (2) and (3) of $C$ we get $\left.\ulcorner\overrightarrow{[x]}]_{\text {germ }_{\mu}}\right\urcorner<y(\omega)$, and then $f\left(\left\ulcorner\overrightarrow{[x]_{\text {germ }_{\mu}}}\right\urcorner\right)<y(\omega)$ by property $(1)$ of $C$. So,

$$
\hat{f}\left(\left\ulcorner\overrightarrow{[x]_{\text {germ }_{\mu}}} \frown[y]_{\mu_{\{\omega\}}}\right\urcorner\right)<\pi_{2}\left(\left\ulcorner\overrightarrow{[x]_{\text {germ }_{\mu}}} \frown[y]_{\mu_{\{\omega\}}}\right\urcorner\right)
$$

and we are done.

We're now able to use the embedding results we have so far to inductively identify the elements of the Kleinberg sequence of the $\omega$-cofinal measure with ultrapowers on $\boldsymbol{\delta}_{2 n+1}^{1}$ :

Corollary 17. There is a canonical measure assignment for $\boldsymbol{\delta}_{2 n+1}^{1}$ and $\mathfrak{A}_{2}$.

Proof. This is a simple inductive argument using Propositions 13 and 16 and Kleinberg's Theorem 3. For details, cf. the argument for $\left(\mathrm{IH}_{*}\right)$ in the proof of Theorem 23.

Corollary 18. If $m<\omega$ and $0<n<\omega$, then the cardinal $\aleph_{\mathbf{e}_{m}+n}$ is Jónsson.

We go on to relate the $\omega$-cofinal measure to the $\omega_{1}$-cofinal measure, proving that the $\omega_{1}$-cofinal measure generates an ultrapower larger than any of the finite iterations of the $\omega$-cofinal measure.

LEMMA 19. Let $\kappa$ be a strong partition cardinal, $\mu$ an order measure on $\kappa$ and $n \in \omega$, then

(1) $\kappa^{\kappa} / \mathcal{C}_{\kappa}^{\omega} \otimes n \leq \kappa^{\kappa} / \mathcal{C}_{\kappa}^{\omega_{1}}$, and

(2) $\kappa^{\kappa} / \mu \oplus \mathcal{C}_{\kappa}^{\omega} \otimes n \leq \kappa^{\kappa} / \mu \oplus \mathcal{C}_{\kappa}^{\omega_{1}}$.

Proof. Since the proof for (1) is nearly identical to that for (2), just slightly simpler, we shall only present a proof for (2). Remember that $\operatorname{germ}_{\mathcal{C}_{\kappa}{ }_{1}}=\mathcal{C}_{\omega_{1}}^{\omega}$ and $\operatorname{germ}_{\mathcal{C}_{\kappa}^{\omega} \otimes n}=\langle\underbrace{\mu_{\{\omega\}}, \ldots, \mu_{\{\omega\}}}_{n}\rangle$. Let $\eta$ be the following measure on $\omega_{1}^{n}:{ }^{[11]}$

$A \in \eta: \Leftrightarrow$ there is a club $C \subseteq \omega_{1} \forall \alpha_{0}<\cdots<\alpha_{n-1} \in C(\vec{\alpha} \in A)$.

Let $m:=\ln _{\mu}$ and define for each $f: \kappa \rightarrow \kappa$ a function $\hat{f}: \kappa \rightarrow \kappa$ by

$$
\left.\left.\left.\hat{f}\left(\ulcorner\vec{\beta}\urcorner[y]_{\operatorname{germ}_{\mathcal{C}_{\kappa} \omega_{1}}}\right\urcorner\right)=\hat{f}\left(\ulcorner\vec{\beta}\urcorner[y]_{\mathcal{C}_{\omega_{1}}^{\omega_{1}}}\right\urcorner\right):=[\vec{\alpha} \mapsto f(\ulcorner\vec{\beta}\urcorner \overrightarrow{y(\alpha)}\urcorner)\right]_{\eta},
$$

where $\vec{\beta} \in[\kappa]^{m}$ and $\overrightarrow{y(\alpha)}$ stands for $\left\langle y\left(\alpha_{0}\right), \ldots, y\left(\alpha_{n-1}\right)\right\rangle$.

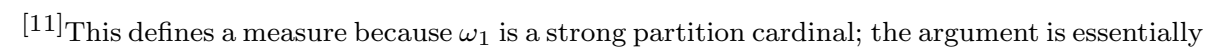
the same as in the Lifting Theorem 10. 
First we have to prove that $\hat{f}$ is welldefined. If $[y]_{\mathcal{C}_{\omega_{1}}}=\left[y^{\prime}\right]_{\mathcal{C}_{\omega_{1}}}$ there is a club set $C \subseteq \omega_{1}$ such that $y(\gamma)=y^{\prime}(\gamma)$ for all $\gamma \in C$, from which it follows immediately that $\left.f(\ulcorner\vec{\beta}\urcorner \overrightarrow{y(\alpha)}\urcorner)=f\left(\ulcorner\vec{\beta}\urcorner \overrightarrow{y^{\prime}(\alpha)}\right\urcorner\right)$ for all $\vec{\alpha} \in[C]^{n}$, i.e., $\left.\hat{f}\left(\ulcorner\vec{\beta}\urcorner[y]_{\mathcal{C}_{\omega_{1}}}\right\urcorner\right)=$ $\left.\hat{f}\left(\ulcorner\vec{\beta}\urcorner\left[y^{\prime}\right]_{\mathcal{C}_{\omega_{1}}}\right\urcorner\right)$.

Now we can show that $f \rightarrow \hat{f}$ induces an embedding from $\kappa^{\kappa} / \mu \oplus \mathcal{C}_{\kappa}^{\omega} \otimes n$ into $\kappa^{\kappa} / \mu \oplus \mathcal{C}_{\kappa}^{\omega_{1}}$. Assume $[f]_{\mu \oplus \mathcal{C}_{\kappa}^{\omega} \otimes n} \leq[g]_{\mu \oplus \mathcal{C}_{\kappa}^{\omega} \otimes n}$, i.e., there is a club $C \subseteq \kappa$ such that for all $\vec{x} \in \mathcal{F}_{\text {o.t. } \mu}^{C}$ and all $\vec{y} \in \mathcal{F}_{\text {o.t. } \mathcal{C}_{\kappa}^{\omega} \otimes n}^{C_{\operatorname{sun}}}$, we get

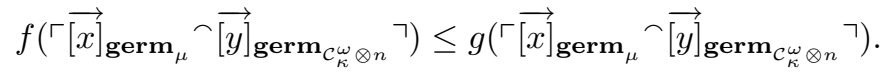

Now because of the peculiar nature of $\operatorname{germ}_{\mathcal{C}_{\kappa}^{\omega} \otimes n}$ which is just $n$ times the principal ultrafilter, we can simplify the $\vec{y}$-part of this expression. For an arbitrary $D \subseteq \kappa$, let $\Omega_{D}:=\left\{\beta\right.$; there is some $z \in[C]^{\omega}$ such that $\left.\beta=\sup z\right\}$. Then

$$
\left.\{\overrightarrow{[y}]_{\operatorname{germ}_{\mathcal{C}_{\kappa} \otimes n}} ; \vec{y} \in \mathcal{F}_{\text {o.t. } \mathcal{C}_{\kappa}^{\mathscr{W}} \otimes n}^{D}\right\}=\left[\Omega_{D}\right]^{n}
$$

With this notation, we get that there is a club $C \subseteq \kappa$ such that for all $\vec{x} \in \mathcal{F}_{\text {o.t. } \mu}^{C}$ and all $\vec{\alpha} \in\left[\Omega_{C_{\text {sup } \vec{x}}}\right]^{n}$, we have

$$
f\left(\left\ulcorner\overrightarrow{[x]} \text { germ }_{\mu} \frown \vec{\alpha}\right\urcorner\right) \leq g\left(\left\ulcorner[\vec{x}]_{\text {germ }_{\mu}} \frown \vec{\alpha}\right\urcorner\right) .
$$

Let $\operatorname{Lim}\left(\omega_{1}\right):=\left\{\beta \in \omega_{1} ; \beta\right.$ is a limit ordinal $\}$. Then for $y \in \mathcal{F}_{\omega_{1}+1}^{C_{\text {sup } \vec{x}}}$ and $\vec{\alpha} \in$ $\left[\operatorname{Lim}\left(\omega_{1}\right)\right]^{n}$ we have $\overrightarrow{y(\alpha)} \in\left[\Omega_{C_{\sup \vec{x}}}\right]^{n}$, so (by the above)

$$
\left.f\left(\left\ulcorner\overrightarrow{[x]} \text { germ }_{\mu} \frown \overrightarrow{y(\alpha)}\right\urcorner\right) \leq g\left(\left\ulcorner\overrightarrow{[x]} \text { germ }_{\mu}\right\urcorner \overrightarrow{y(\alpha)}\right\urcorner\right) .
$$

This means that for all $\vec{x} \in \mathcal{F}_{\text {o.t. } \mu}^{C}$ and $y \in \mathcal{F}_{\omega_{1}+1}^{C_{\text {sup } \vec{x}}}$ exists a club set $C^{\prime}:=\operatorname{Lim}\left(\omega_{1}\right) \subseteq$ $\omega_{1}$ such that

$$
\left.\left.\forall \alpha_{0}<\cdots<\alpha_{n-1} \in C^{\prime} f\left(\left\ulcorner[\overrightarrow{x]}]_{\operatorname{germ}_{\mu}}\right\urcorner \overrightarrow{y(\alpha)}\right\urcorner\right) \leq g\left(\left\ulcorner[\overrightarrow{[x]}]_{\text {germ }_{\mu}}\right\urcorner \overrightarrow{y(\alpha)}\right\urcorner\right),
$$

i.e., by definition of $\eta$,

$$
\left.\left.\left.\left[\vec{\alpha} \mapsto f\left(\ulcorner\overrightarrow{[x]}]_{\operatorname{germ}_{\mu}}\right\urcorner \overrightarrow{y(\alpha)}\right\urcorner\right)\right]_{\eta} \leq\left[\vec{\alpha} \mapsto g\left(\left\ulcorner[\overrightarrow{x]}]_{\operatorname{germ}_{\mu}}\right\urcorner \overrightarrow{y(\alpha)}\right\urcorner\right)\right]_{\eta} .
$$

But this means $[\hat{f}]_{\mu \oplus \mathcal{C}_{\kappa}^{\omega_{1}}} \leq[\hat{g}]_{\mu \oplus \mathcal{C}_{\kappa}^{\omega_{1}}}$.

Lemma 20. Let $\omega<\kappa<\Theta$ be a regular cardinal and $\mu$ a measure on $\kappa$. Then $\kappa^{\kappa} / \mu$ has cofinality greater $\omega$.

Proof. Let $\left\langle\alpha_{i} ; i \in \omega\right\rangle$ be a sequence in $\kappa^{\kappa} / \mu$. Lemma 4 allows us to pick a sequence $\left\langle f_{i} ; i \in \omega\right\rangle \in\left(\kappa^{\kappa}\right)^{\omega}$ such that $\left[f_{i}\right]_{\mu}=\alpha_{i}$ for $i \in \omega$. For $\xi \in \kappa$ let $g(\xi):=\sup _{i \in \omega} f_{i}(\xi)$. Since $\kappa>\omega$ is regular, we have $g \in \kappa^{\kappa}$ and for all $i \in \omega$, we have $\left[f_{i}\right]_{\mu} \leq[g]_{\mu} \in \kappa^{\kappa} / \mu$, so the sequence $\left\langle\alpha_{i} ; i \in \omega\right\rangle$ cannot be cofinal in $\kappa^{\kappa} / \mu$.

Proposition 21. Let $\kappa$ be a strong partition cardinal, $\mu$ an order measure on $\kappa$ and $n \in \omega$, then

$$
\left(\kappa^{\kappa} / \mu\right)^{(\omega+1)} \leq \kappa^{\kappa} /\left(\mu \oplus \mathcal{C}_{\kappa}^{\omega_{1}}\right) .
$$


Proof. By repeatedly applying Proposition 16 we get that for any $n \in \omega$

$$
\left(\kappa^{\kappa} / \mu\right)^{(n)} \leq \kappa^{\kappa} /\left(\mu \oplus \mathcal{C}_{\kappa}^{\omega} \otimes n\right) .
$$

By taking the supremum over $n$ on both sides and using Lemma 19 this yields

$$
\left(\kappa^{\kappa} / \mu\right)^{(\omega)} \leq \sup _{n \in \omega}\left(\kappa^{\kappa} / \mu \oplus \mathcal{C}_{\kappa}^{\omega} \otimes n\right) \leq \kappa^{\kappa} / \mu \oplus \mathcal{C}_{\kappa}^{\omega_{1}}
$$

but we know from Lemma 20 that $\operatorname{cf}\left(\kappa^{\kappa} / \mu \oplus \mathcal{C}_{\kappa}^{\omega_{1}}\right)>\omega$, so

$$
\left(\kappa^{\kappa} / \mu\right)^{(\omega)}<\kappa^{\kappa} /\left(\mu \oplus \mathcal{C}_{\kappa}^{\omega_{1}}\right) \text {. }
$$

\section{An abstract combinatorial computation}

In our computation we shall use two main tools, the first is the following theorem which is an elaboration of the proof of the "moreover" part in Theorem 3:

TheOREm 22 (Ultrapower Shifting Lemma). Let $\beta$ and $\gamma$ be ordinals and let $\mu$ be a $\kappa$-complete ultrafilter on $\kappa$ with $\kappa^{\kappa} / \mu=\kappa^{(\gamma)}$. If for all cardinals $\kappa<\nu \leq \kappa^{(\beta)}$

- either $\nu$ is a successor and $\operatorname{cf}(\nu)>\kappa$,

- or $\nu$ is a limit and $\operatorname{cf}(\nu)<\kappa$, then $\left(\kappa^{(\beta)}\right)^{\kappa} / \mu \leq \kappa^{(\gamma+\beta)}$.

Proof. Cf. [Lö02, Lemma 2.7].

THEOREM 23. Let $\kappa$ be a strong partition cardinal and assume $\kappa^{\kappa} / \mathcal{C}_{\kappa}^{\omega}=\kappa^{+}$ and $\kappa^{\kappa} / \mathcal{C}_{\kappa}^{\omega_{1}}=\kappa^{(\omega+1)}$.

Then for all $\xi<\omega^{2}$, the following equalities hold:

- $\left(\kappa^{(\xi)}\right)^{\kappa} / \mathcal{C}_{\kappa}^{\omega_{1}}=\kappa^{(\omega+1+\xi)}$.

- if $1+\xi=\omega \cdot m+n$ then

$$
\begin{gathered}
\kappa^{(\xi+1)}=\kappa^{\kappa} /\left(\mathcal{C}_{\kappa}^{\omega_{1}} \otimes m \oplus \mathcal{C}_{\kappa}^{\omega} \otimes n\right)= \\
=\left(\ldots \left(\left((\ldots(\kappa^{\kappa} / \underbrace{\left.\left.\mathcal{C}_{\kappa}^{\omega}\right)^{\kappa} / \ldots\right)^{\kappa} / \mathcal{C}_{\kappa}^{\omega}}_{n})^{\kappa} / \underbrace{\left.\left.\mathcal{C}_{\kappa}^{\omega_{1}}\right)^{\kappa} / \ldots\right)^{\kappa} / \mathcal{C}_{\kappa}^{\omega_{1}}}_{m}\right.\right.\right.
\end{gathered}
$$

- $\operatorname{cf}\left(\kappa^{(\xi+1)}\right)=\left\{\begin{array}{cl}\kappa^{+} & \text {if } \xi \text { is a successor or zero, or } \\ \kappa^{(\omega+1)} & \text { if } \xi>0 \text { is a limit. }\end{array}\right.$

- $\operatorname{cf}\left(\kappa^{(\xi)}\right)= \begin{cases}\kappa & \text { if } \xi=0, \text { or } \\ \omega & \text { if } \xi>0 \text { is a limit. }\end{cases}$

ProOF. By assumption $\kappa$ is a strong partition cardinal, thus regular. Also, for all limit ordinals $\xi<\omega^{2}$, the cofinality of $\kappa^{(\xi)}$ is $\omega$. So the last part of our theorem is trivial.

By Kleinberg's Theorem 3 (1), (2) and (5), we have for $n \in \omega$ that $\operatorname{cf}\left(\kappa^{(n+1)}\right)=$ $\kappa^{+}\left(\dagger_{1}\right)$. We'll now prove by induction that for $n>0$, we have

$$
\kappa^{(n)}=\kappa^{\kappa} / \mathcal{C}_{\kappa}^{\omega} \otimes n=\left(\ldots(\kappa^{\kappa} / \underbrace{\left.\left.\mathcal{C}_{\kappa}^{\omega}\right)^{\kappa} / \ldots\right)^{\kappa} / \mathcal{C}_{\kappa}^{\omega}}_{n} \quad\left(\dagger_{2}\right):\right.
$$

By assumption $\kappa^{+}=\kappa^{\kappa} / \mathcal{C}_{\kappa}^{\omega}$, so the statement is true for $n=1$. Assume the statement holds for $n$, then 


$$
\begin{array}{rlr}
\kappa^{(n+1)} & =\left(\kappa^{(n)}\right)^{+}=\left(\kappa^{\kappa} / \mathcal{C}_{\kappa}^{\omega} \otimes n\right)^{+} & \text {(Induction hypothesis) } \\
& \leq \kappa^{\kappa} / \mathcal{C}_{\kappa}^{\omega} \otimes(n+1) & \text { (Proposition 16) } \\
\leq\left(\kappa^{\kappa} / \mathcal{C}_{\kappa}^{\omega} \otimes n\right)^{\kappa} / \mathcal{C}_{\kappa}^{\omega} & \text { (Lemma 13) } \\
& \left.=\kappa^{(n)}\right)^{\kappa} / \mathcal{C}_{\kappa}^{\omega} \leq \kappa^{(n+1)} & \text { (Induction hypothesis) } \\
& \leq \kappa^{(n+1)} . & \text { (Lemma 22) }
\end{array}
$$

We denote the conjunction of $\left(\dagger_{1}\right)$ and $\left(\dagger_{2}\right)$ by $\left(\mathrm{IH}_{*}\right)$.

We proceed by induction on $\xi$, using the following induction hypothesis: ${ }^{[12]}$

$\left(\mathrm{IH}_{\xi}\right)\left[\begin{array}{l}\text { For all } \alpha \leq \xi, \text { the following three conditions hold: } \\ \text { 1. }\left(\kappa^{(\alpha)}\right)^{\kappa} / \mathcal{C}_{\kappa}^{\omega_{1}}=\kappa^{(\omega+1+\alpha)}, \\ \text { 2. If } \alpha=\beta+1 \text { is a successor and } 1+\beta=\omega \cdot m+n \text { then } \\ \left(\kappa^{(\alpha)}\right)^{\kappa} / \mathcal{C}_{\kappa}^{\omega_{1}}=\kappa^{\kappa} / \mathcal{C}_{\kappa}^{\omega_{1}} \otimes(m+1) \oplus \mathcal{C}_{\kappa}^{\omega} \otimes n=\left(\kappa^{\kappa} / \mathcal{C}_{\kappa}^{\omega_{1}} \otimes m \oplus \mathcal{C}_{\kappa}^{\omega} \otimes n\right)^{\kappa} / \mathcal{C}_{\kappa}^{\omega_{1}} \\ \text { 3. } \operatorname{cf}\left(\kappa^{(\omega+1+\alpha)}\right):=\left\{\begin{array}{cl}\omega & \text { if } \alpha>0 \text { is a limit, } \\ \kappa^{+} & \text {if } \alpha \text { is } 1 \text { or a double successor, or } \\ \kappa^{(\omega+1)} & \text { if } \alpha \neq 1 \text { is zero or a single } \\ & \text { successor. }\end{array}\right.\end{array}\right.$

Obviously, if $\left(\mathrm{IH}_{*}\right)$ and all $\left(\mathrm{IH}_{\xi}\right)$ (for $\left.\xi<\omega^{2}\right)$ hold, the theorem is proven.

By assumption, $\left(\kappa^{(0)}\right)^{\kappa} / \mathcal{C}_{\kappa}^{\omega_{1}}=\kappa^{\kappa} / \mathcal{C}_{\kappa}^{\omega_{1}}=\kappa^{(\omega+1)}$ and from Theorem $3(1)$, we know that this is a regular cardinal, so $\left(\mathrm{IH}_{0}\right)$ holds.

For the successor step $\xi \mapsto \xi+1$ assume that $\left(\mathrm{IH}_{\xi}\right)$ holds. We first prove parts 1. and 2. of $\left(\mathrm{IH}_{\xi+1}\right)$. Since $\xi+1$ is a successor ordinal, $1+\xi=\omega \cdot m+n$ holds, where $m, n \in \omega$ and not both are zero. We consider the three cases:

Case 1: $m=0$ and $n>0$, i.e., $\xi+1=i+1, i \in \omega$. We have to prove

$$
\kappa^{(\omega+1+i+1)}=\left(\kappa^{(i+1)}\right)^{\kappa} / \mathcal{C}_{\kappa}^{\omega_{1}}=\kappa^{\kappa} / \mathcal{C}_{\kappa}^{\omega_{1}} \oplus \mathcal{C}_{\kappa}^{\omega} \otimes(i+1)=\left(\kappa^{\kappa} / \mathcal{C}_{\kappa}^{\omega} \otimes(i+1)\right)^{\kappa} / \mathcal{C}_{\kappa}^{\omega_{1}},
$$

which we shall do by induction.

For $i=0$ we have

$$
\begin{array}{rlr}
\kappa^{(\omega+1+1)}=\left(\kappa^{(\omega+1)}\right)^{+} & =\left(\kappa^{\kappa} / \mathcal{C}_{\kappa}^{\omega_{1}}\right)^{+} & \text {(Assumption) } \\
& \leq \kappa^{\kappa} / \mathcal{C}_{\kappa}^{\omega_{1}} \oplus \mathcal{C}_{\kappa}^{\omega} & \text { (Proposition 16) } \\
& \leq\left(\kappa^{\kappa} / \mathcal{C}_{\kappa}^{\omega}\right)^{\kappa} / \mathcal{C}_{\kappa}^{\omega_{1}} & \text { (Lemma 13) } \\
& =\left(\kappa^{+}\right)^{\kappa} / \mathcal{C}_{\kappa}^{\omega_{1}} & \text { (Assumption) } \\
& \leq \kappa^{(\omega+1+1)} . & \text { (Lemma 22) }
\end{array}
$$

Assume the statement holds for $i$, then

$$
\begin{aligned}
\kappa^{(\omega+1+(i+1)+1)} & =\left(\kappa^{(\omega+1+i+1)}\right)^{+} \\
& =\left(\kappa^{\kappa} / \mathcal{C}_{\kappa}^{\omega_{1}} \oplus \mathcal{C}_{\kappa}^{\omega} \otimes(i+1)\right)^{+} \\
& \leq \kappa^{\kappa} / \mathcal{C}_{\kappa}^{\omega_{1}} \oplus \mathcal{C}_{\kappa}^{\omega} \otimes(i+1+1) \\
& \leq\left(\kappa^{\kappa} / \mathcal{C}_{\kappa}^{\omega} \otimes(i+1+1)\right)^{\kappa} / \mathcal{C}_{\kappa}^{\omega_{1}} \\
& =\left(\kappa^{((i+1)+1)}\right)^{\kappa} / \mathcal{C}_{\kappa}^{\omega_{1}} \\
& \leq \kappa^{(\omega+1+(i+1)+1)} .
\end{aligned}
$$

Case 2: $m>0$ and $n=0$, i.e., $\xi+1=\omega \cdot m+1$. We have to prove $\kappa^{(\omega+1+\omega \cdot m+1)}=\left(\kappa^{(\omega \cdot m+1)}\right)^{\kappa} / \mathcal{C}_{\kappa}^{\omega_{1}}=\kappa^{\kappa} / \mathcal{C}_{\kappa}^{\omega_{1}} \otimes(m+1)=\left(\kappa^{\kappa} / \mathcal{C}_{\kappa}^{\omega_{1}} \otimes(m)\right)^{\kappa} / \mathcal{C}_{\kappa}^{\omega_{1}}$,

${ }^{[12]}$ An ordinal $\gamma$ is a double successor is there is some $\delta$ such that $\gamma=\delta+2$. An ordinal is a single successor if it's a successor but not a double successor; equivalently, if it is the successor of a limit ordinal. 
which we shall do as usual by induction.

For $m=1$ we have

$$
\begin{array}{rlr}
\kappa^{(\omega+1+\omega+1)}=\left(\kappa^{(\omega+1)}\right)^{(\omega+1)} & =\left(\kappa^{\kappa} / \mathcal{C}_{\kappa}^{\omega_{1}}\right)^{(\omega+1)} & \text { (Assumption) } \\
& \leq \kappa^{\kappa} / \mathcal{C}^{\omega_{1}} \oplus \mathcal{C}_{\kappa}^{\omega_{1}} & \text { (Proposition 21) } \\
\leq\left(\kappa^{\kappa} / \mathcal{C}_{\kappa}^{\omega_{1}}\right)^{\kappa} / \mathcal{C}_{\kappa}^{\omega_{1}} & \text { (Lemma 13) } \\
& =\left(\kappa^{(\omega+1)}\right)^{\kappa} / \mathcal{C}_{\kappa}^{\omega_{1}} & \text { (Assumption) } \\
& \leq \kappa^{(\omega+1+\omega+1)} . & \text { (Lemma 22) }
\end{array}
$$

Assume the statement holds for $m$, then

$$
\begin{array}{rlr}
\kappa^{(\omega+1+\omega \cdot(m+1)+1)} & =\left(\kappa^{(\omega+1+\omega \cdot m+1)}\right)^{(\omega+1)} & \\
& =\left(\kappa^{\kappa} / \mathcal{C}_{\kappa}^{\omega_{1}} \otimes(m+1)\right)^{(\omega+1)} & \\
& \leq \kappa^{\kappa} / \mathcal{C}_{\kappa}^{\omega_{1}} \otimes(m+1+1) & \text { (Induction hypothesis) } \\
& \leq\left(\kappa^{\kappa} / \mathcal{C}_{\kappa}^{\omega_{1}} \otimes(m+1)\right)^{\kappa} / \mathcal{C}_{\kappa}^{\omega_{1}} & \text { (Proposition 21) } \\
& =\left(\kappa^{(\omega+1+\omega \cdot m+1)}\right)^{\kappa} / \mathcal{C}_{\kappa}^{\omega_{1}} & \text { (Induction hypothesis) } \\
& \leq \kappa^{(\omega+1+\omega \cdot(m+1)+1)} . & \text { (Lemma 22) }
\end{array}
$$

Case 3: $m>0$ and $n>0$, i.e., $\xi+1=\omega \cdot m+n+1$. We have to prove

$$
\begin{aligned}
& \left(\kappa^{(\omega \cdot m+n+1)}\right)^{\kappa} / \mathcal{C}_{\kappa}^{\omega_{1}}=\kappa^{\kappa} / \mathcal{C}_{\kappa}^{\omega_{1}} \otimes(m+1) \oplus \mathcal{C}_{\kappa}^{\omega} \otimes(n+1) \\
= & \left(\kappa^{\kappa} / \mathcal{C}_{\kappa}^{\omega_{1}} \otimes m \oplus \mathcal{C}_{\kappa}^{\omega} \otimes(n+1)\right)^{\kappa} / \mathcal{C}_{\kappa}^{\omega_{1}}=\kappa^{(\omega+1+\omega \cdot m+n+1)} .
\end{aligned}
$$

Assuming the statement holds for $\xi=\omega \cdot m+n$, then

$$
\begin{array}{rlr}
\kappa^{(\omega+1+\omega \cdot m+n+1)} & =\left(\kappa^{(\omega+1+\omega \cdot m+1)}\right)^{(n)} & \\
& =\left(\kappa^{\kappa} / \mathcal{C}_{\kappa}^{\omega_{1}} \otimes(m+1)\right)^{(n)} & \\
& \leq\left(\kappa^{\kappa} / \mathcal{C}_{\kappa}^{\omega_{1}} \otimes(m+1) \oplus \mathcal{C}_{\kappa}^{\omega}\right)^{(n-1)} & \\
& \vdots & \\
& \leq \kappa^{\kappa} / \mathcal{C}_{\kappa}^{\omega_{1}} \otimes(m+1) \oplus \mathcal{C}_{\kappa}^{\omega} \otimes n & \\
& \leq\left(\kappa^{\kappa} / \mathcal{C}_{\kappa}^{\omega_{1}} \otimes m+\oplus \mathcal{C}_{\kappa}^{\omega} \otimes n\right)^{\kappa} / \mathcal{C}_{\kappa}^{\omega_{1}} & \\
& =\left(\kappa^{(\omega+1+\omega \cdot(m-1)+n)}\right)^{\kappa} / \mathcal{C}_{\kappa}^{\omega_{1}} & (\text { Proposition 16) } \\
& \leq \kappa^{(\omega+1+\omega \cdot m+n+1)} . & (\text { Lemma 13) } \\
& & \left(\text { IH }_{\xi}\right) \\
& &
\end{array}
$$

We shall now compute the cofinality of $\kappa^{(\omega+1+(\xi+1))}$ in order to check that part 3. of $\left(\mathrm{IH}_{\xi+1}\right)$ holds:

Case 1: $\xi<\omega$. In this case, $\operatorname{cf}\left(\kappa^{(\xi+1)}\right)=\kappa^{+}>\kappa$ by $\left(\mathrm{IH}_{*}\right)$. So, we can apply Lemma 6 to $\lambda:=\kappa^{(\xi+1)}$. Thus

$$
\begin{aligned}
\operatorname{cf}\left(\kappa^{(\omega+1+(\xi+1))}\right) & =\operatorname{cf}\left(\left(\kappa^{(\xi+1)}\right)^{\kappa} / \mathcal{C}_{\kappa}^{\omega_{1}}\right) \\
& =\operatorname{cf}\left(\kappa^{(\xi+1)}\right) \\
& =\kappa^{+} .
\end{aligned}
$$

Case 2: $\omega \leq \xi<\omega^{2}$. In this case, there is an ordinal $\alpha<\xi$ such that $\xi+1=\omega+1+\alpha$, and the following equivalences hold:

$(*)\left[\begin{array}{rl}\alpha \text { is } 1 \text { or a double successor } & \Longleftrightarrow \xi \text { is a successor }, \\ \alpha \neq 1 \text { is zero or a single successor } & \Longleftrightarrow \xi \text { is a limit. }\end{array}\right.$ 
Now, by $\left(\mathrm{IH}_{\xi}\right)$, we get that $\operatorname{cf}\left(\kappa^{(\xi+1)}\right)=\operatorname{cf}\left(\kappa^{(\omega+1+\alpha)}\right)>\kappa$. So, again applying Lemma 6 to $\lambda:=\kappa^{(\xi+1)}$, we get

$$
\begin{aligned}
\operatorname{cf}\left(\kappa^{(\omega+1+(\xi+1))}\right) & =\operatorname{cf}\left(\left(\kappa^{(\xi+1)}\right)^{\kappa} / \mathcal{C}_{\kappa}^{\omega_{1}}\right) \\
& =\operatorname{cf}\left(\kappa^{(\xi+1)}\right) \\
& =\operatorname{cf}\left(\kappa^{(\omega+1+\alpha)}\right),
\end{aligned}
$$

thus by $(*)$

$$
\operatorname{cf}\left(\kappa^{(\omega+1+(\xi+1))}\right)=\left\{\begin{array}{cl}
\kappa^{+} & \text {if } \xi \text { is a successor, and } \\
\kappa^{(\omega+1)} & \text { if } \xi \text { is a limit. }
\end{array}\right.
$$

This ends the proof of the induction step for $\xi \mapsto \xi+1$.

Note that for the limit step $\xi=\lambda$ we only have to check parts 1 . and 3 . of $\left(\mathrm{IH}_{\lambda}\right)$. Let $0<\lambda<\omega^{2}$ be a limit ordinal. This implies that for some $\alpha<\lambda$, we have that $\omega+\alpha=\lambda$. We now assume $\left(\mathrm{IH}_{\eta}\right)$ for $\eta<\lambda$, and write $\left(\mathrm{IH}_{<\lambda}\right)$ for this assumption. In particular (since $\alpha<\lambda$ ), we know the cofinalities of all cardinals between $\kappa$ and $\kappa^{(\omega+1+\alpha)} \geq \kappa^{(\omega+\alpha)}=\kappa^{(\lambda)}$. This allows us to apply the Ultrapower Shifting Lemma 22 for $\gamma=\omega+1$ and $\beta=\lambda$ :

$$
\begin{array}{rlr}
\sup \left\{\kappa^{(\omega+1+\eta)} ; \eta<\lambda\right\} & =\sup \left\{\left(\kappa^{(\eta)}\right)^{\kappa} / \mathcal{C}_{\kappa}^{\omega_{1}} ; \eta<\lambda\right\} & \left(\mathrm{IH}_{<\lambda}\right) \\
& \leq\left(\kappa^{(\lambda)}\right)^{\kappa} / \mathcal{C}_{\kappa}^{\omega_{1}} & \\
& \leq \kappa^{(\omega+1+\lambda)} & \\
& =\sup \left\{\kappa^{(\omega+1+\eta)} ; \eta<\lambda\right\} . &
\end{array}
$$

This establishes $\left(\kappa^{(\lambda)}\right)^{\kappa} / \mathcal{C}_{\kappa}^{\omega_{1}}=\kappa^{(\omega+1+\lambda)}$. The claim about the cofinality of $\kappa^{(\omega+1+\lambda)}$ is trivial for a limit ordinal $\lambda<\omega^{2}$. So $\left(\mathrm{IH}_{\lambda}\right)$ holds and we are finished with our induction.

\section{Applications of the inductive analysis}

We shall now rephrase Theorem 23 in terms of measure assignments and get computations of further Kleinberg sequences as an application.

\subsection{Application 1: Measure Analysis.}

COROLlary 24. Let $n>0$. Then there is a canonical measure assignment for $\boldsymbol{\delta}_{2 n+1}^{1}$ and $\mathfrak{A}_{3}^{\oplus}$.

Proof. Let $\kappa:=\boldsymbol{\delta}_{2 n+1}^{1}$ and let $\mathrm{V}_{0}$ and $\mathrm{V}_{1}$ be the generators of $\mathfrak{A}_{3}^{\oplus}$. We let $\operatorname{meas}\left(\mathrm{V}_{0}\right):=\mathcal{C}_{\kappa}^{\omega}$ and meas $\left(\mathrm{V}_{1}\right):=\mathcal{C}_{\kappa}^{\omega_{1}}$. By Theorem $5(7)$, we have that $\kappa^{\kappa} / \mathcal{C}_{\kappa}^{\omega}=\kappa^{+}$. Furthermore, it is well-known that $\kappa^{\kappa} / \mathcal{C}_{\kappa}^{\omega_{1}}=\kappa^{(\omega+1)}$ (a lower bound is given by our Proposition 21, an upper bound requires some further computation). Therefore, $\kappa$ satisfies the assumptions of Theorem 23 with $\mu_{0}=\mathcal{C}_{\kappa}^{\omega}$ and $\mu_{1}=\mathcal{C}_{\kappa}^{\omega_{1}}$ which says that the measure assignment is canonical. ${ }^{[13]}$

Corollary 25. Let $n>0$ and $m \in \omega$. Then $\aleph_{\mathbf{e}_{n}+\omega \cdot m+1}$ is Jónsson, and $\aleph_{\mathbf{e}_{n}+\omega^{2}}$ is Rowbottom.

${ }^{[13]}$ Strictly speaking, we should still have to show that measure addition respects ordinal addition. For instance, since $o\left(\mathrm{~V}_{0}+\mathrm{V}_{1}\right)=1+\omega=o\left(\mathrm{~V}_{1}\right)$, we should have that the measures $\mathcal{C}_{\kappa}^{\omega} \oplus \mathcal{C}_{\kappa}^{\omega_{1}}$ and $\mathcal{C}_{\kappa}^{\omega_{1}}$ give the same ultrapower. We leave this as an exercise for the reader who can follow the proof idea of Proposition 19. 
PRoOF. As in the proof of Corollary 24, we apply Theorem 23 to $\kappa:=\boldsymbol{\delta}_{2 n+1}^{1}=$ $\aleph_{\mathbf{e}_{n}+1}$ and the measures $\mu_{0}:=\mathcal{C}_{\kappa}^{\omega}$ and $\mu_{1}:=\mathcal{C}_{\kappa}^{\omega_{1}}$. Let $\kappa_{m}:=\kappa_{m}^{\mu_{1}}$ be the elements of the Kleinberg sequence derived from $\mu_{1}$, i.e., $\kappa_{m+1}=\left(\kappa_{m}\right)^{\kappa} / \mu_{1}$. Theorem 23 now immediately gives us the computation of the $\kappa_{m}$ as:

$$
\kappa_{m+1}=\aleph_{\mathbf{e}_{n}+\omega \cdot m+1} .
$$

Now the claim follows directly from Kleinberg's Theorem 3.

Note that Corollary 25 also gives a computation of the Jónsson cardinals $\aleph_{\omega \cdot m+1}$ that were computed in [Lö02, Corollary 3.3], however, -as opposed to the proof in [Lö02] - the proof given here does not refer to the full Jackson-Khafizov analysis of ultrapowers.

4.2. Application 2: Some more Jónsson cardinals. Let us give another computation of more Jónsson cardinals that resolves an open question from [Lö02]: We mentioned in $[\mathbf{L o ̈ 0 2}, \S 4]$ that $\aleph_{\omega \cdot 2+2}$ is "the first infinite cardinal of which we do not know whether it has any large cardinal properties under AD". We shall now show that it is a Jónsson cardinal. The proof is a straightforward analysis of material that can be found in Kleinberg's book [Kl77].

TheOREM 26 (Kleinberg). If $\kappa \rightarrow(\kappa)^{\kappa}, \mu$ is a normal measure on $\kappa, \lambda \rightarrow$ $(\lambda)_{\kappa^{2}}^{\kappa+\kappa}$, and $\xi:=\lambda^{\kappa} / \mu$, then we have

$$
\xi \rightarrow[\xi]_{\kappa,<\aleph_{0}}^{<\omega}
$$

Proof. [K177, Theorem 4.9]

LEMma 27. Let $\xi$ be a cardinal such that $\xi \rightarrow[\xi]_{\kappa,<\aleph_{0}}^{<\omega}$ for some $\aleph_{1} \leq \kappa<\xi$. Then $\xi$ is a Jónsson cardinal.

Proof. Let $F:[\xi]^{<\omega} \rightarrow \xi$ be a colouring. Let $s: \xi \rightarrow \kappa$ be a surjection. Define a new colouring $G:=s \circ F$. By assumption, we have some $C \in[\xi]^{\xi}$ such that $G\left[[C]^{n}\right]$ is countable for each $n \in \mathbb{N}$. Let $\alpha \in \kappa$ be such that $\alpha$ is not hit by $G$, and let $\beta \in \xi$ be such that $s(\beta)=\alpha$. Then $\beta \notin F\left[[C]^{<\omega}\right]$. This shows $\xi \rightarrow[\xi]_{\xi}^{<\omega}$.

Theorem 28. For every $n<\omega$, the cardinal $\aleph_{\mathbf{e}_{n}+\omega+2}$ is Jónsson.

Proof. Let $\kappa, \mu_{0}$ and $\mu_{1}$ as in the proof of Corollaries 24 and 25. Then $\lambda:=\kappa^{\kappa} / \mu_{0}=\delta_{2 n+2}^{1}$ which satisfies the partition relation $\lambda \rightarrow(\lambda)^{\alpha}$ for all $\alpha<\lambda$ by Theorem 3 . We can again apply Theorem 23 and get an ultrapower representation:

$$
\aleph_{\mathbf{e}_{n}+\omega+2}=\kappa^{\kappa} /\left(\mu_{1} \oplus \mu_{0}\right)=\left(\kappa^{\kappa} / \mu_{0}\right)^{\kappa} / \mu_{1} \text {. }
$$

The partition relation yields $\lambda \rightarrow(\lambda)_{\kappa^{2}}^{\kappa+\kappa}$, and thus $\aleph_{\mathbf{e}_{n}+\omega+1}:=\lambda^{\kappa} / \mu_{1}$ is Jónsson by Theorem 26 and Lemma 27 .

Theorem 28 shifts the questionable honour of being "the first infinite cardinal of which we do not know whether it has any large cardinal properties under AD" from $\aleph_{\omega \cdot 2+2}$ to $\aleph_{\omega \cdot 2+3}$. A proof that $\aleph_{\omega \cdot 2+3}$, and in general $\aleph_{\omega \cdot 2+n}$ is Jónsson seems to be well in reach of the current methods. Let us define a quasi-Kleinberg sequences by $\kappa_{1}^{\mathrm{Q}}:=\kappa, \kappa_{2}^{\mathrm{Q}}:=\kappa^{\kappa} / \mu_{0}$, and $\kappa_{n+1}^{\mathrm{Q}}:=\left(\kappa_{n}^{\mathrm{Q}}\right)^{\kappa} / \mu_{1}$ for $n \geq 2$. A Kleinberg theorem (analogous to Theorem 3) for quasi-Kleinberg sequences would yield the Jónsson property for the cardinals $\aleph_{\omega \cdot 2+n}$. 


\section{References}

[Co96] Eoin Coleman, Jónsson groups, rings and algebras, Bulletin of the Irish Mathematical Society 36 (1996), p. 34-45

[Ja88] Steve Jackson, AD and the Projective Ordinals, in: A. S. Kechris, D. A. Martin, J. R. Steel (eds.), Cabal Seminar 81-85, Proceedings, Caltech-UCLA Logic Seminar 1981-85, Springer-Verlag 1988 [Lecture Notes in Mathematics 1333], p. 117-220

[Ja99] Steve Jackson, A Computation of $\boldsymbol{\delta}_{5}^{1}$, Memoirs of the American Mathematical Society 140 (1999), viii+94 pages

[JaKh $\infty$ ] Steve Jackson, Farid T. Khafizov, Descriptions and Cardinals below $\boldsymbol{\delta}_{5}^{1}$, accepted for publication in Journal of Symbolic Logic

[JaLö $\infty]$ Steve Jackson, Benedikt Löwe, Canonical Measure Assignments. in preparation

[Ka94] Akihiro Kanamori, The Higher Infinite, Large Cardinals in Set Theory from Their Beginnings, Springer-Verlag 1994 [Perspectives in Mathematical Logic]

[Ke78] Alexander S. Kechris, AD and Projective Ordinals, in: A.S.Kechris, Y. N. Moschovakis, Cabal Seminar 76-77, Proceedings, Caltech-UCLA Logic Seminar 1976-77, Springer-Verlag 1978 [Lecture Notes in Mathematics 689], p. 91-132

[K177] Eugene M. Kleinberg, Infinitary Combinatorics and the Axiom of Determinateness, Springer-Verlag 1977 [Lecture Notes in Mathematics 612]

[Lö02] Benedikt Löwe, Kleinberg Sequences and partition cardinals below $\boldsymbol{\delta}_{5}^{1}$, Fundamenta Mathematicae 171 (2002), p. 69-76.

(S. Bold \& B. Löwe) Institute for Logic, Language and Computation, Universiteit van Amsterdam, Plantage Muidergracht 24, 1018 TV Amsterdam, The Netherlands

E-mail address: $\{$ sbold, bloewe\}@science.uva.nl

(S. Bold \& B. Löwe) Mathematisches Institut, Rheinische Friedrich-Wilhelms-UniverSität Bonn, Beringstrasse 1, 53115 Bonn, Germany

E-mail address: $\{$ bold, loewe\}@math.uni-bonn.de

(B. Löwe) Fachbereich Mathematik, Universität Hamburg, Bundesstrasse 55, 20146 HAMBURG, GERMANY

E-mail address: loewe@math.uni-hamburg.de 